\title{
Trois disques d'Europe orientale (Roumanie, Transylvanie)
}

\section{Etienne Bours}

\section{(2) OpenEdition}

\section{Journals}

Édition électronique

URL : http://journals.openedition.org/ethnomusicologie/212

ISSN : 2235-7688

Éditeur

ADEM - Ateliers d'ethnomusicologie

Édition imprimée

Date de publication : 1 décembre 2001

Pagination : 333-335

ISBN : 2-8257-07-61-9

ISSN : 1662-372X

\section{Référence électronique}

Etienne Bours, «Trois disques d'Europe orientale (Roumanie, Transylvanie)», Cahiers

d'ethnomusicologie [En ligne], 14 | 2001, mis en ligne le 10 janvier 2012, consulté le 05 mai 2019. URL http://journals.openedition.org/ethnomusicologie/212

Ce document a été généré automatiquement le 5 mai 2019.

Tous droits réservés 


\title{
Trois disques d'Europe orientale (Roumanie, Transylvanie)
}

\author{
Etienne Bours
}

\section{RÉFÉRENCE}

Fin de millénaire dans le village roumain. Enregistrements (1989-1997) : Speran†a Rădulescu, Jacques Bouët, Bernard Lortat-Jacob, Adriana Dan. Sélection de la musique et texte (roumain) : Speranta Rădulescu. Texte (français) : Jacques Bouët. Texte (anglais) : Margaret Beissinger. Production : Fundatia Al. Tzigara-Samurcas, Collection Ethnophonie. 1 CD CD001.

Fanfares paysannes de Moldavie : Zece Prajini. Enregistrements (1997): Speranta \& Valeriu Rădulescu. Sélection de la musique et texte : Speran†a Rădulescu. Version française : Annie Bentoiu \& Jacques Bouët. Version anglaise : Adrian Solomon \& Dan Bora. Production : Fundatia Al. Tzigara-Samurcas, Collection Ethnophonie. 1 CD CD002. Musiques hongroises de Transylvanie. Traditions du Gyimes et de la Grande Plaine. Enregistrements (1997-2000) : studios Fono Records de Budapest. Direction de collection, sélection des plages et texte : Laszlo Kelemen et Robert Kerényi. Traduction (français et anglais) : Dorothée Kovácsházy. 1 CD Fono Records - Inédit, Maison des Cultures du Monde W260098.

1 Ces trois disques compacts ont un certain nombre de points communs. Le premier est de faire découvrir des musiques de villages de Roumanie, Moldavie et Transylvanie. Le second est la démarche profonde, engagée, des chercheurs et producteurs qui, d'un côté comme de l'autre, animés d'une volonté farouche de ne pas perdre l'essentiel, prolongent un siècle d'enregistrements ethnographiques. L'esprit de Bartók souffle sur ces trois disques. On se souvient que, vers 1907, le compositeur était contrarié par le fait que nombre de chanteurs et musiciens de villages oubliaient leurs traditions au profit d'airs à la mode venus des villes. Bartók appelait à la vigilance, disant haut et fort que les musiques des villages vivaient peut-être là leur dernière heure. Plus tard, il fit d'ailleurs remarquer que les travaux des Roumains (guidés par Constantin Brailoiu) pouvaient être 
utiles aux Hongrois, rappelant que la Roumanie avait développé dès 1934 une collection d'enregistrements de musiques paysannes sur disque Gramophone alors que la Hongrie n'en possédait encore aucun. Dans la foulée, les disques Patria remédièrent à cette lacune avec une série de disques hongrois qui furent mis sur le marché en 1939. Bartók voyait en cette collection la concrétisation d'une de ses volontés: «sauvegarder les chants populaires hongrois de style ancien et surtout enregistrer sur disques l'interprétation paysanne originale de ces chants» (Hungarian Folk Music Hungaroton LPX18058-60, notice intérieure).

2 On serait sans doute tenté de dire que, depuis lors, tout a été mis en œuvre pour continuer la démarche de Bartók et que, plus que jamais, les musiques villageoises de Hongrie et de Roumanie jouissent d'une certaine reconnaissance. On pense au mouvement de renaissance qui emboîta le pas au chercheur et à ses émules, Kodály en tête ; on pense au mouvement de danses Tanchaz, aux nombreux collectages effectués par les musiciens eux-mêmes, à l'avènement d'une nouvelle génération de musiciens très familiers de ces musiques. On pense aux nombreux disques plus ou moins accessibles, aux archives de plus en plus ouvertes, aux concerts... Mais ce serait aller un peu vite en besogne que de croire, avec un optimisme béat, que ces musiques villageoises ne connaîtront jamais leur dernière heure. Et ces trois nouveaux disques sont là pour nous rappeler qu'aujourd'hui encore la même vigilance s'impose, d'autant plus que les dérives se multiplient.

3 En Hongrie, Fono Music Hall a créé en 1997 le programme "La dernière heure ", vaste entreprise d'enregistrements tous azimuts des groupes villageois de Transylvanie. En 1998, Fono Records a débuté la publication d'une série de disques compacts intitulée « Uj Patria », qui sont autant de sélections parmi ces enregistrements. Soit une immense opération qui prolonge celles qu'avaient présidées Bartók, Kodály et Laszlo Lajtha dans les années 1930. Inédit travaille en collaboration avec Fono, éditant des enregistrements de cette série à l'occasion de la venue de musiciens hongrois de Transylvanie au Festival de l'Imaginaire de 2001. Soit un duo de musiciens Csangos de la vallée de la Tatros, jouant violon et gardon pour un répertoire de danses hongroises et complaintes chantées, et un taraf tsigane jouant sur violons, altos et contrebasse des musiques et chants liés à la vie quotidienne du village. Un disque qui nous ramène aux musiques plus rudes des campagnes, loin du peaufinement, parfois magnifique au demeurant, des musiciens $d u$ revival.

Ces musiques du quotidien sont celles que les deux disques roumains entendent aussi privilégier. Des disques dont la production relève d'une démarche conjointe entre Musée du Paysan, Ministère de la Culture et Fond culturel européen, démarche prouvant une fois de plus cette volonté de sauvegarder des musiques qui pourraient encore et toujours en être à leur dernière heure. Pour s'en convaincre, il suffit de comparer les notices des deux livrets signées par Speranła Rădulescu avec ce que la même chercheuse écrivait dans le vol. 13 des Cahiers de musiques traditionnelles sur les métissages "pan-balkaniques » en Roumanie. Imperceptiblement peut-être, du moins de loin, les paysages musicaux de ces pays et de ces régions changent sans cesse, reléguant les musiques les plus rurales aux oubliettes ou, en tous cas, dans le sac des expressions déconsidérées. Pourtant, ces disques nous apportent un éclairage exceptionnel sur ces musiques qu'on a l'impression de bien connaitre parce qu'on a tendance à écouter un certain nombre de disques sans nécessairement relever la date d'enregistrement. A ce titre, il est du plus haut intérêt de comparer ces deux disques actuels avec le $\mathrm{CD}$ récemment sorti dans la série Rounder « 
World Library of Folk and Primitive Music ", nous redonnant des enregistrements de musiques roumaines effectués entre 1934 et 1957 et publiés dans la fameuse série de Lomax vers 1960 (Rounder CD 1759). Une comparaison rassurante quant à l'état de ces musiques hier mais aujourd'hui aussi !

Des deux disques ici présentés le premier propose des musiques calendaires s'échelonnant au fil des douze mois de l'année, entre chansons lyriques, danses jouées par des fanfares ou des taraf, chants rituels, musiques à écouter et pièces « solitaires ». L'autre volume explique et démontre avec perfection le phénomène des fanfares paysannes de Moldavie. Le livret y est remarquable, tant par son explication historique que par son analyse actuelle. Les enregistrements sont de qualité et ces deux disques, comme celui d'Inédit pour la Transylvanie, ont le mérite immense de nous rappeler l'anonymat médiatique de ces musiques qui continuent d'évoluer dans la discrétion de leur quotidien. Elles qui, pourtant, sont souvent véhiculées, parfois adroitement, parfois déformées, par le biais de musiciens professionnels auxquels le marché des musiques du monde s'est ouvert. La source de ces derniers n'est guère tarie, comme nous le montrent ces trois disques qui sont, en même temps, un plaidoyer pour qu'on ne la laisse pas tarir et une abondante matière à réflexion sur l'utilisation des musiques populaires. Et aussi la preuve que chercheurs, amateurs, musiciens, collecteurs et autres, ont prolongé la dernière heure de ces musiques depuis déjà un siècle. 